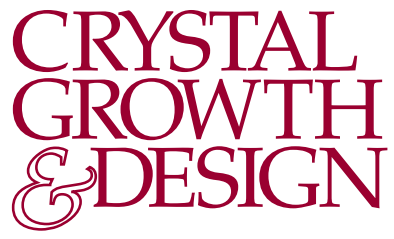

\title{
Impact of Mixed Solvent on Co-Crystal Solubility, Ternary Phase Diagram, and Crystallization Scale Up
}

\author{
${ }_{3}$ Tasnim Munshi, ${ }^{, \dagger}$ Batul Redha, ${ }^{\dagger}$ Neil Feeder, ${ }^{,}$Paul Meenan, ${ }^{\S}$ and Nicholas Blagden $"$ \\ $4{ }^{\dagger}$ School of Chemistry, University of Lincoln, Lincoln, U.K. LN6 7TS \\ 5 ॠCambridge Crystallographic Data Centre, 12 Union Road, Cambridge, U.K. \\ $6{ }^{\S}$ Pfizer Global Research \& Development, Pharmaceutical Sciences, Sandwich, U.K. and Pharmaceutical Sciences, Pfizer Global R \& D, \\ 7 Groton, Connecticut 06340, United States \\ ${ }_{8}$ "School of Pharmacy, University of Lincoln, Lincoln, U.K. LN6 7TS
}

10 ABSTRACT: This contribution covers the identification, understanding, and rationale of the interplay between the choice of mixed solvent on the crystallization of the co-crystal system benzoic acid and isonicotinamide (BZ:INA). A critical first step was gauging the impact of solvent choice and composition on the overall crystallization process, across a number of temperature points. This required defining the solubility and phase diagrams of the co-crystal system at specified temperatures, which reflects the cooling crystallization profile encountered in a batch crystallization step. To this end, identifying and understanding the impact of solvent composition over a selected temperature range on the solubility of co-crystal underpins this contribution.

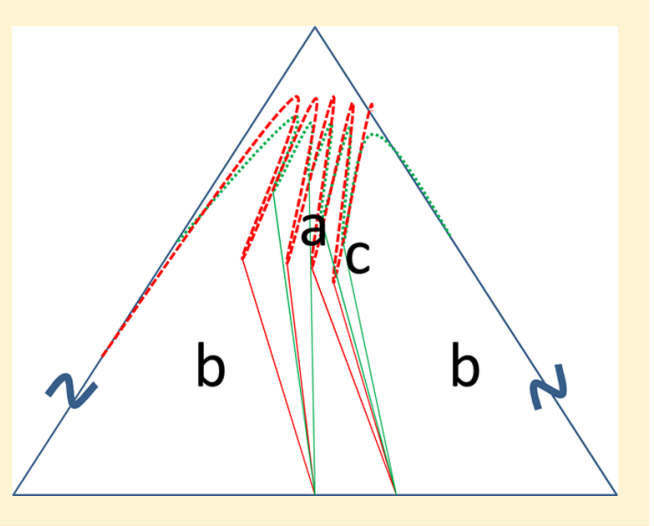

\section{INTRODUCTION}

21 Significant literature exists on the design and synthesis of co22 crystals and the application of co-crystals in drug development. ${ }^{1}$ 23 However, the focus of this contribution centers on the area of 24 bulk crystallization and the scale up of the solution 25 crystallization route. This aspect of co-crystal research is 26 presently an unexplored area, but critical insight is required if 27 co-crystals are to be routinely isolated from solution and used 28 in the fine chemical and pharmaceutical sectors.

29 The motivation for considering the application of co-crystals 30 as a dosage form stems from the need to generate stable 31 crystalline material in order to have selectivity and control over 32 the chemical and physical properties of drug entities, ${ }^{1}$ 33 specifically, increasing the solubility and manipulating the 34 dissolution profile in order to improve bioavailability. Allied to 35 this is the notion that the solubility of the drug is related to 36 drug absorption, and in the crystallization process there is a 37 need to have knowledge of the solubility profiles and many 38 studies had been carried out and reported. ${ }^{2}$ These have focused 39 on the trends of polymorph solubility, dissolution and 40 thermodynamic behavior, and the impact of thermodynamic 41 and kinetic factors on the formation of different polymorphs. ${ }^{3}$ 42 This study is the natural progression of these types of concepts 43 for polymorphic systems to co-crystal systems.

44 The purpose of this work is to examine the role of solvent 45 and a mixed solvent composition on the solution co46 crystallisation process. We have chosen the isonicotinamide 47 (INA) and benzoic acid (BA) co-crystal system, as much literature exists in the determination of co-crystal growth from 48 these two compounds in a single solvent and the impact of 49 component ratio through complex formation of the 1:1 and 2:1 50 complexes. ${ }^{4,5}$ It has been previously reported how differences in 51 solubility of the compounds map onto the profile of a typical 52 ternary phase diagram for a 1:1 co-crystal, with compounds 53 with different solubility mapping to the formation of a skewed 54 phase diagram. ${ }^{6}$ We report here a more complex situation 55 where a co-crystal has both a 1:1 and 2:1 complex, when a 56 mixed system is employed, and will examine the impact of this 57 in designing the co-crystallisation isolation step.

We report specifically on the impact of a (i) mixed solvent 59 through manipulating the solubility of coformers and molecular 60 complexes and (ii) the impact of composition on the 61 compounds as defined by the ternary phase diagram and how 62 these contribute to the design of the crystallization for this class 63 of compounds.

The solubility of co-crystals in a 1:1 and 2:1 composition in 65 the mixed solvents was analyzed and determined by a single 66 mathematical equation, and data were fitted to the cosolvency 67 model of the general single model (GSM). ${ }^{7}$ In order to 68 determine the thermodynamic factors that control the 69 formation of these molecular systems, the change in enthalpy 70

Received: June 30, 2015

Revised: February 17, 2016 
71 and entropy in solution was determined using the van't Hoff 72 equation.

\section{MATERIALS AND METHODS}

74 For further details on the experimental details, see Supporting 75 Information and the Redhas thesis. ${ }^{8}$

76 All chemicals were purchased from Sigma-Aldrich in the highest 77 purity and were used as supplied.

78 Co-crystals were grown in water, ethanol, and an ethanol/water 79 solvent (30-90\% ethanol). The products were characterized by 80 powder X-ray diffraction (PXRD), Raman, infrared, and ${ }^{1} \mathrm{H}$ NMR 81 spectroscopy.

82 Solubility. The Jouyban-Acree model was used to predict the 83 solubility of co-crystal, ${ }^{8-11}$ and the solubility was also determined 84 experimentally (see Supporting Information).

85 X-ray Powder Diffraction. Samples were analyzed using a Bruker $86 \mathrm{D} 8$ diffractometer (wavelength of X-ray $0.154 \mathrm{~nm} \mathrm{Cu}$ source). The 87 solids were scanned from $5-50^{\circ}$, with a 0.01 step width and $1 \mathrm{~s}$ time 88 count. The receiving slit was $1^{\circ}$, and the scatter slit was $0.2^{\circ}$.

89 Solubility Determination. The React-Array Microvate (low 90 throughput) was used to determine the solubility. The samples were 91 placed in glass tubes, and the solutions were stirred at temperatures of 9225,35 , and $40{ }^{\circ} \mathrm{C}$, the samples were held at these temperatures for 80 $93 \mathrm{~h}$, and then the solubility was determined gravimetrically.

94 The predicted solubility in the mixed solvent was calculated using 95 the Jouyban-Acree model, and the deviation from the experimental 96 solubility was determined. ${ }^{12,13}$

97 Phase Diagram. The screen method developed by Blagden and 98 Boyed et al. was used for this study., ${ }^{5}$ The RUMED incubator was 99 used to incubate the slurry solution for 2 weeks, and the phases were 100 determined with PXRD.

101 The ternary phase diagram was constructed at 20 and $40{ }^{\circ} \mathrm{C}$, in $50 \%$ 102 ethanol, and the solids were analyzed using PXRD. These ternary 103 phase diagram was plotted using the ProSim software ternary diagram 104 plot. $^{16}$

105 The phase diagrams, along with the solubility at 50\% ethanol 106 solution composition, were subsequently employed to design the 107 cooling crystallization at $100 \mathrm{~cm}^{3}$ volume.

\section{RESULTS AND DISCUSSION}

109 Solubility. First, the solubility of benzoic acid, isonicotina110 mide, and $1: 1$ and 2:1 co-crystals at 25 and $40{ }^{\circ} \mathrm{C}$ was 111 determined in water, ethanol, and a water/ethanol mixed 112 solvent (30-90\% ethanol), and the change in the solubility was 113 observed. Second, for completeness the direct solubility of the 114 co-crystals and coformers in a mixed solvent was also analyzed, 115 and a hyperbolic solubility profile was observed; the values in 116 pure solvent agree with those previously reported. ${ }^{5}$

117 In order to undertake the van't Hoff equation, an analysis of 118 the solubility curves of the co-crystal was redrawn such that In 119 solubility vs 1 /temp was plotted, and the profiles for solubility 120 are given in Figure 1a and Figure $1 \mathrm{~b}$ for the 1:1 and 2:1 co121 crystals, respectively (see Supporting Information).

Solubility Behavior of Co-Crystal Coformers in a 123 Mixed Solvent System. The solubility behavior of the 1:1 124 and 2:1 co-crystals was examined as the water to ethanol 125 content was varied. For both co-crystals, the solubility was at a 126 minimum in water and increased as the solvent composition 127 approached $70 \% \mathrm{v} / \mathrm{v}$ and decreased as the ethanol composition 128 increased. The trend was highly distinctive for the $2: 1$ co-crystal 129 system (Figure 2).

130 The trends in molar enthalpy and entropy for both the 1:1 131 and 2:1 co-crystal systems (Figure 2) are similar for both 132 systems, and the enthalpy is consistent and does not change as 133 the ethanol concentration is increased; however, a notable 134 variation is seen as the concentration of ethanol is increased.

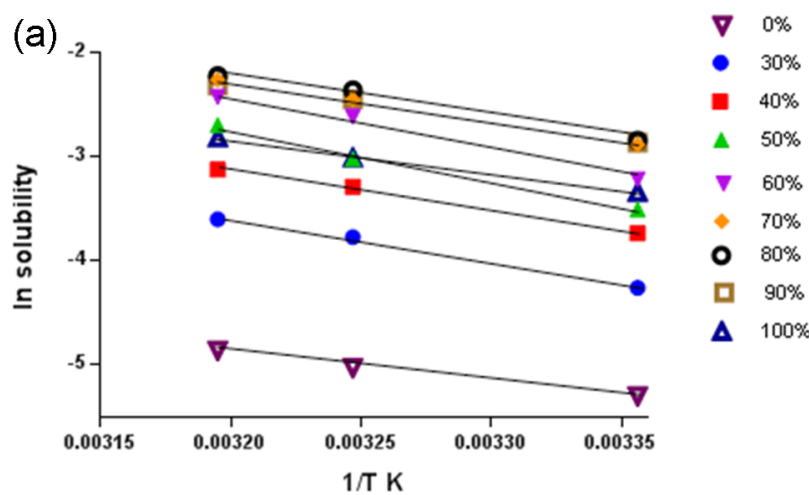

(b)

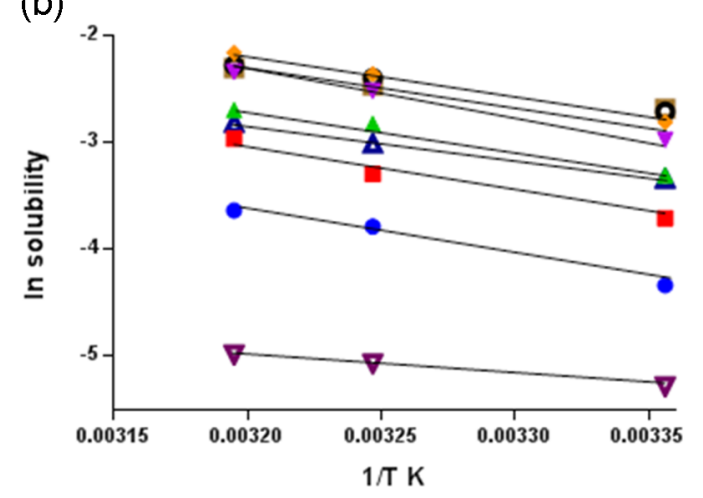

$\nabla 0 \%$

- $30 \%$

- $40 \%$

A $50 \%$

v $60 \%$

- $70 \%$

- $80 \%$

ㅁ. $90 \%$

A $100 \%$

Figure 1. (a) Change in the solubility of co-crystal (1:1) with the inverse of the change of temperature. (b) Change in the solubility of co-crystal (2:1) with the inverse of the change of temperature.

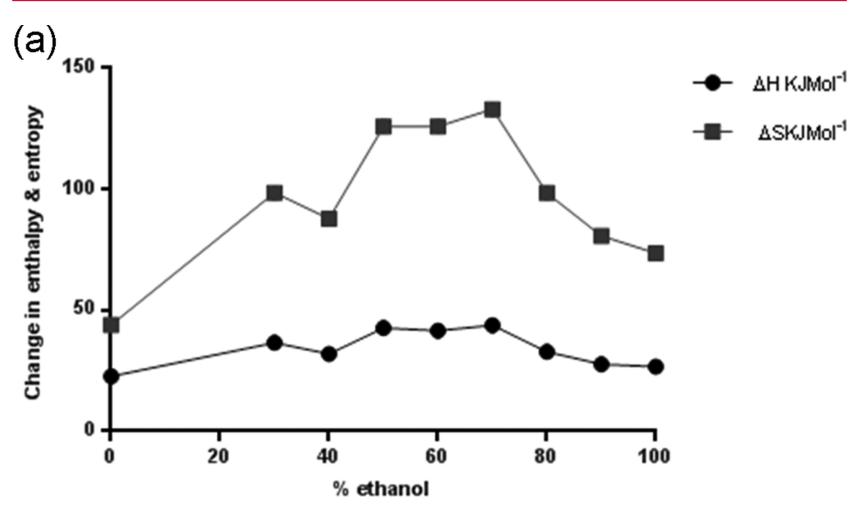

(b)

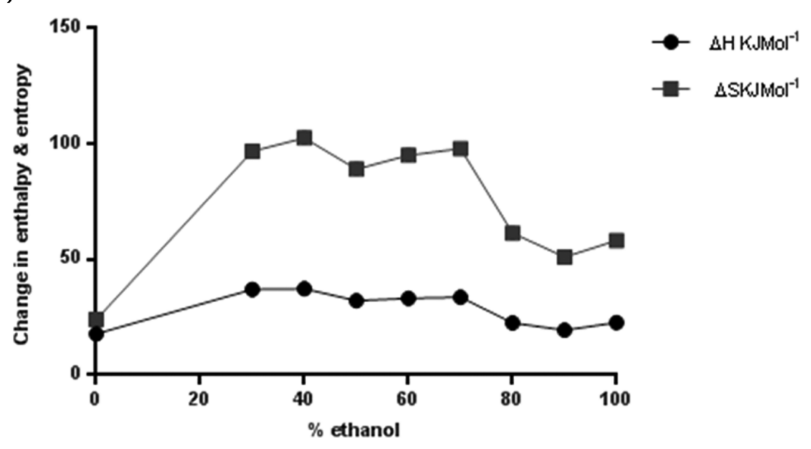

Figure 2. (a) Change in $\Delta H$ molar enthalpy of solution, $\Delta S$ molar entropy of solution of co-crystals $1: 1$ in solution. (b) Change in $\Delta H$ the molar enthalpy of solution, $\Delta S$ the molar entropy of solution of cocrystals 2:1 in solution. 
135 The implication of these profiles is that the mode of assembly 136 (e.g., hydrogen bond usage and solute solvent interaction) is 137 consistent between the levels of association, i.e., monomers, 138 dimers, chains, etc.; however, the level of assembly varies with 139 regard to the solvation cage and molecular association. This 140 inference is consistent, with a similar mode assembly between 141 the 1:1 and 1:2 occurring in the solution, mirroring the mode of 142 assembly in the crystal structures, in that the enthalpy has a 143 similar but less marked behavior to entropy.

144 Another approach to the solubility trends is to model 145 solubility in a mixed solvent. A current model used to 146 determine solubility in mixed solvents is the Jouyban-Acree 147 model. $^{7}$ A generic model based on the Jouyban-Acree model 148 was developed by training using the literature data for an 149 ethanol/water mixed solvent (see Supporting Information). 150 The model gives poor results for this system because of the two 151 coformers, which overcomplicates the system.

152 Summary of Solubility Trends. The deviation of the 153 actual solubility from the ideal solubility may indicate that there 154 is a solute-solvent interaction. This suggests that it is critical to 155 obtain verification of nonideal contribution, which may be 156 achieved by examining the deviation from idealities of the 157 measured solubility's $(W)$ to those of the calculated ideal $(Q)$ at 158 a specific temperature (deviation taken as $W / Q T=$ solubility in 159 mixed solvent/log-linear model at specific temperatures, see 160 Supporting Information).

161 For this reason the deviation in actual solubility from the 162 ideal solubility obtained by the ratio of experimental solubility 163 with the ideal solubility calculated using solvent fraction and 164 solute coformer solubility in the respective pure solvents was 165 examined at $25{ }^{\circ} \mathrm{C}$ (blue), $35{ }^{\circ} \mathrm{C}$ (red), and $40{ }^{\circ} \mathrm{C}$ (green) 166 Figure $3 \mathrm{a}(1: 1)$ and Figure $3 \mathrm{~b}(2: 1)$.

167 This approach clearly verifies the observations seen with the 168 solubility fitting, whereby two distinct variable solutions
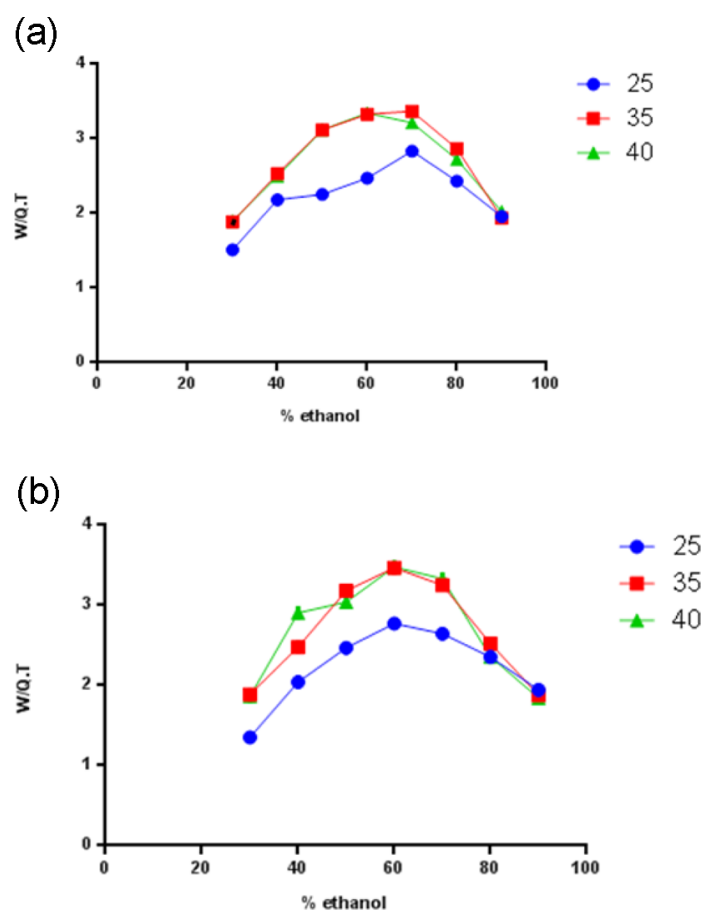

Figure 3. (a) Deviation of the solubility of co-crystals (1:1) in the mixed solvent from ideal solubility. (b) Deviation of the solubility of co-crystals $(2: 1)$ in the mixed solvent from ideal solubility. regimes exist, and these are dependent on temperature, 169 irrespective of the stoichiometric ratio. This type of trend 170 also suggests that the water breaks up the association and thus 171 affects the balance of monomers versus dimers, etc., as does 172 increasing the temperature, and this is supported by the profiles 173 obtained as the amount of water is increased (Figure 3). 174

Optimising the Solution Crystallization Conditions. 175 The phase diagrams and subsequent crystallization studies 176 focused on the 40 to $20{ }^{\circ} \mathrm{C}$ temperature range, and these were 177 undertaken in an ethanol-water composition determined by 178 the solubility studies; all solutions prior to crystallization were 179 saturated at $40{ }^{\circ} \mathrm{C}$ and taken to $50{ }^{\circ} \mathrm{C}$ to ensure complete 180 dissolution. The clearest way to extract the initial solvent 181 composition point was to examine the solubility profiles and to 182 confirm the \% composition point, for this work 50\% was 183 identified. This was undertaken in order to maximize the 184 hypothetical yield, which for our purposes was the highest 185 possible amount of material in solution, and typically this was 186 around $45-55 \%$. From this starting point, two linked studies 187 were undertaken, (i) slow cooling from $50{ }^{\circ} \mathrm{C}$ at a rate of 1 to 188 $0.5{ }^{\circ} \mathrm{C}$, to determine the point at which crystallization occurs; 189 this is taken as the under cooling point for our purposes, 190 Figures 4a and 5a, and (ii) step cooling crystallizations from $50191 \mathrm{f} 4 \mathrm{f5}$ ${ }^{\circ} \mathrm{C}$ to the identified under cooling point, to determine the time 192 for crystallization to occur; which relates to the induction time, 193 Figures $4 \mathrm{~b}$ and $5 \mathrm{~b}$. This is taken as the induction time for 194 crystallization for our purposes.

For the 1:1 system, the curve profile shows that the 196 temperature was at its lowest when the co-crystals were grown 197
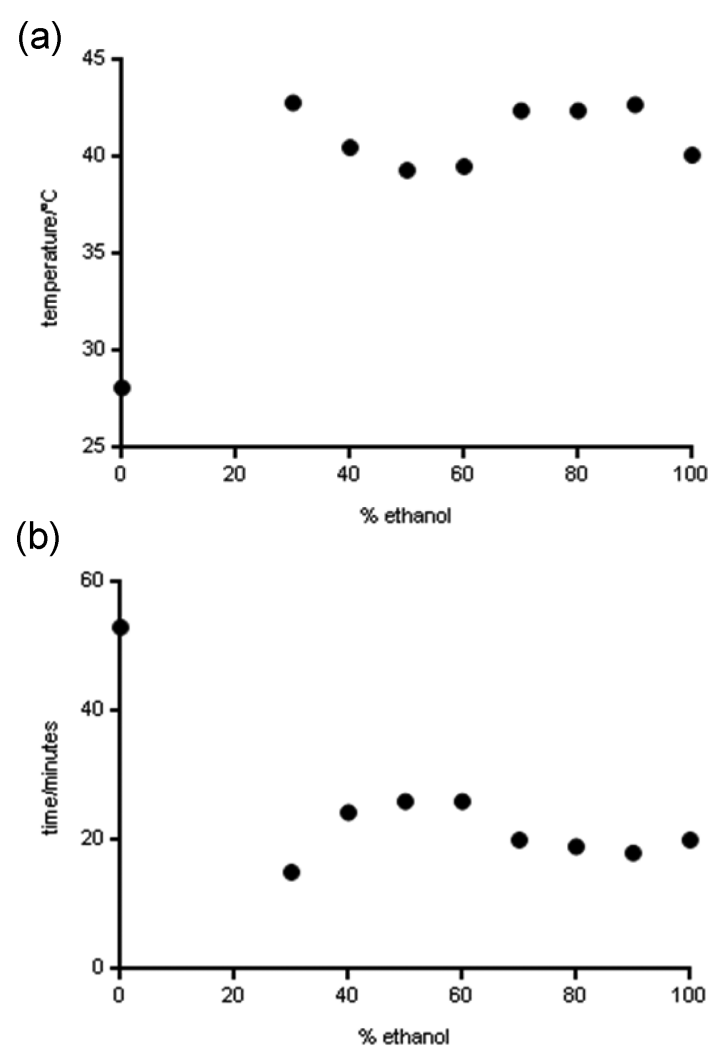

Figure 4. (a) Onset crystallization temperature, relates to the undercooling from $50{ }^{\circ} \mathrm{C}$, BZ:INA (1:1) in water, ethanol, and ethanol/water mixture. (b) Induction time required to start crystallization from the undercooling point from BZ:INA (1:1) in water, ethanol, and ethanol/water mixture. 
(a)

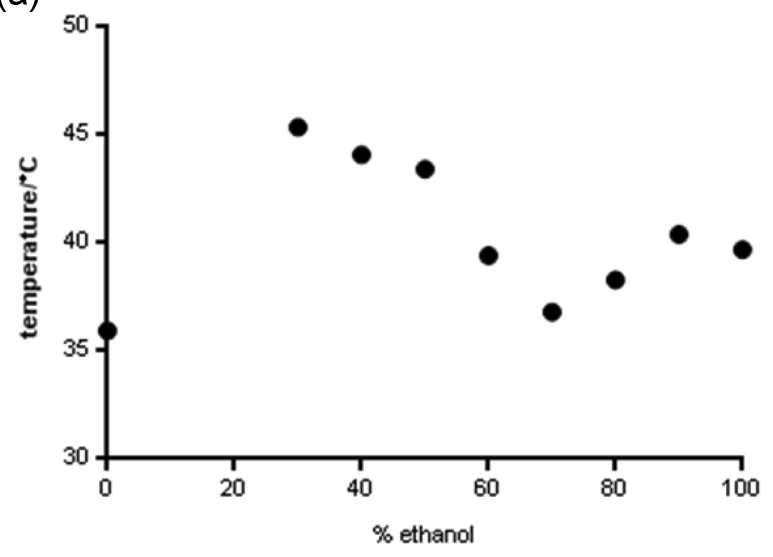

(b)

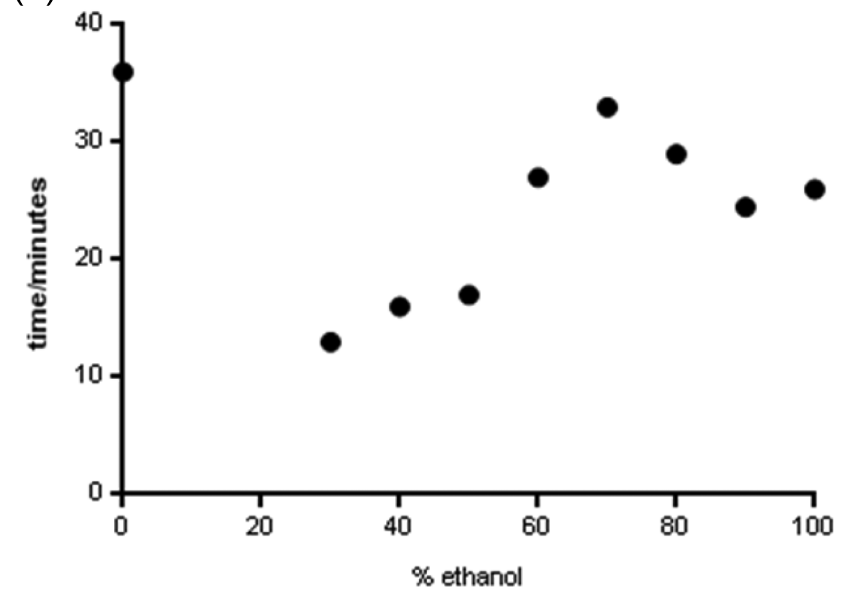

Figure 5. (a) Onset crystallization temperature relates to the undercooling from $50{ }^{\circ} \mathrm{C}$ of BZ:INA (2:1) in water, ethanol, and ethanol-water mixture. (b) Induction time required to start crystallization from the undercooling point from of BZ:INA $(2: 1)$ in water, ethanol, and ethanol-water mixture.

198 in water at $28^{\circ} \mathrm{C}$, as the concentration of ethanol was increased 199 the temperature increased sharply in the 30\% ethanol solvent 200 and reached $42.8^{\circ} \mathrm{C}$, while the temperature fluctuated between $20139.3-42.8^{\circ} \mathrm{C}$ at higher ethanol concentration. The maximum 202 time for the start of crystallization in water was $53 \mathrm{~min}$ (Figure 2034 ), and the time in 30\% ethanol-water mixture was $15 \mathrm{~min}$. 204 The time for the onset of crystallization was found to have 205 increased to $26 \mathrm{~min}$ at $50-60 \%$ ethanol, and it fluctuated as the 206 concentration of ethanol was increased.

207 For the 2:1 system the curve profile shows that the 208 crystallization onset temperature in water was low $\left(36{ }^{\circ} \mathrm{C}\right)$, 209 and the temperature increased to $45.4{ }^{\circ} \mathrm{C}$ in $30 \%$ ethanol, and 210 the temperature dropped to $36{ }^{\circ} \mathrm{C}$ in the $70 \%$ ethanol solvent 211 and fluctuated as the concentration of ethanol was increased. 212 The curve profile for the induction time for the 2:1 co-crystal 213 indicates that the maximum time required for the start of 214 crystallization in water was $39 \mathrm{~min}$, the time dropped sharply to $21515 \mathrm{~min}$ in the $30 \%$ ethanol mixture, and the time of 216 crystallization increased to $30 \mathrm{~min}$ at $50-60 \%$ ethanol 217 concentration and decreased further as the concentration of 218 ethanol was increased to around $22 \mathrm{~min}$.

219 These overall sets of undercooling and induction time data 220 indicate that in pure water the crystallization process would proceed more rapidly than at other solution compositions, and 221 with less under cooling. In addition, the 1:1 system at greater 222 than $50 \%$ ethanol appears to behave consistently, whereas the 223 2:1 system over a similar composition range appears to go 224 through a maximum and then decrease over the same 225 composition range. This may be a facet of a kinetic of the 226 crystallization process of this system, and therefore the phase 227 diagram for this system was re-evaluated at $20^{\circ} \mathrm{C}$ and also at 40228 ${ }^{\circ} \mathrm{C}$. These two phase diagrams were then taken as the 229 thermodynamic start and end point of the step cooling 230 crystallization process.

The Ternary Phase Diagram at 20 and $40^{\circ} \mathrm{C}$. The phase 232 diagram at 20 and $40{ }^{\circ} \mathrm{C}$ shows a skewed profile at both 233 temperatures, which reflects the differences in the solubility in 234 $50 \%$ ethanol of benzoic acid and isonicotinamide. Similar work 235 carried out by Seaton et al..$^{5}$ in pure solvents at $25{ }^{\circ} \mathrm{C}$ showed 236 that the phase diagram in the water system was heavily skewed, 237 but both $1: 1$ and 1:2 phases can be grown, while in the ethanol 238 system only co-crystals 1:1 were grown and was less skewed. 239

The solubility within the phase diagrams at the two 240 temperatures defines the start and end point for a temperature 241 drop cooling crystallization. To link the two isothermal points 242 of the phase diagrams with thermodynamic phase equilibrium 243 points during the step crystallization process, it is important to 244 recognize the deviation in the solubility line between the two 245 temperatures, in defining the labile region during the step 246 cooling process which drives supersaturation. This schematic 247 was derived from overlaying the two phase diagram with the 248 scaling focused upon the eutectic/solubility regions over the 249 region which defines 1:1 only, 1:1 concurrent with 2:1 and 2:1 250 only. Figure 6 highlights the viable labile region for the $251 \mathrm{f} 6$

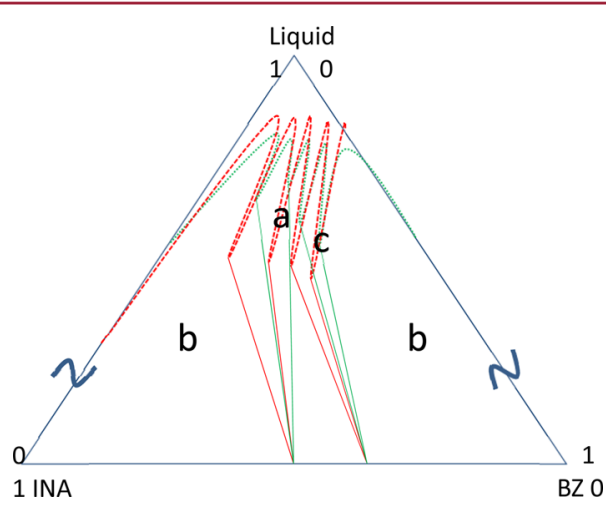

Figure 6. This schematic highlights the deviation between the ternary phase diagrams at $20^{\circ} \mathrm{C}$ (dash lines) and $40{ }^{\circ} \mathrm{C}$ (solid line). (a) For $1: 1$, (b) concurrent region, and (c) for $2: 1$.

crystallization from $40{ }^{\circ} \mathrm{C}$ (solid) to $20{ }^{\circ} \mathrm{C}$ (dash) for specific 252 solid phase compositions, marked (a) for 1:1, (b) concurrent 253 region, and (c) for 2:1 respectively.

This approach was taken to specify the crystallization 255 conditions in which the system was driven through the labile 256 region at $50{ }^{\circ} \mathrm{C}$, through the phase equilibrium at $40{ }^{\circ} \mathrm{C}$ and 257 onto phase equilibrium at $20^{\circ} \mathrm{C}$; these two latter temperature 258 points define the thermal separation employed for these 259 studies. Selection was based upon creating a cooling profile 260 with composition which would establish conditions for 261 crystallization in which one co-crystal phase relation to another 262 could be examined. ${ }^{16}$ With regard to the phase diagrams, 263 cooling a solution below its liquidus line at tie line requires 264 refined screening to define the transition from one crystal 265 
266 composition to another in order to define the tie line 267 boundary $^{17}$ and defines the regions on concurrent phase 268 formation. Thus, it is important to use the full set of 269 constructed ternary phase diagrams at various solution 270 compositions to identify the optimum cooling crystallization.

Solution Crystallization - 1:1 to 2:1 Interconversions 272 and Role of Seeding. The details of deviation in the 273 solubility, tie lines, size, and position of the different regions 274 and the eutectic points are presented in Figures 7 and 8. The

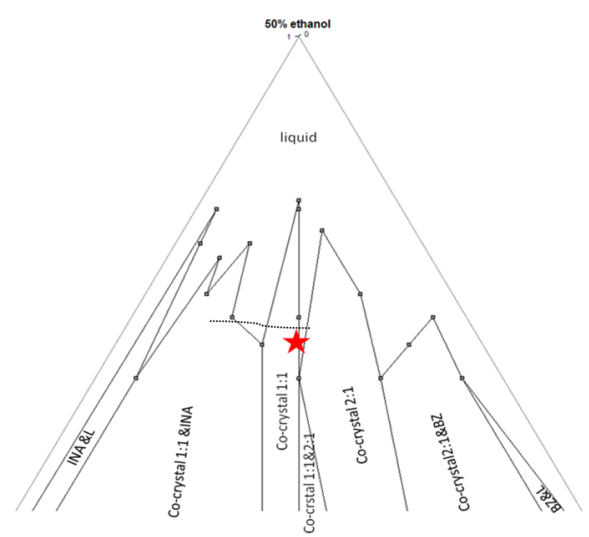

At 40 degree

Figure 7. Upper part of the ternary phase diagram of benzoic acid, isonicotinamide, and $50 \%$ ethanol at $40{ }^{\circ} \mathrm{C}$ (the red point shows the composition of the mixture used in this experiment).

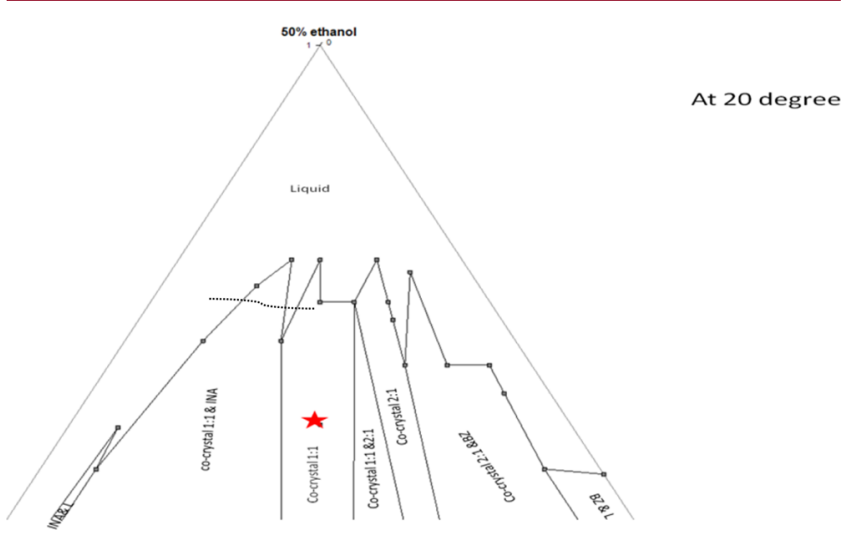

Figure 8. Upper part of the ternary phase diagram of benzoic acid, isonicotinamide, and $50 \%$ ethanol at $20{ }^{\circ} \mathrm{C}$ (the red point shows the same composition of the mixture used at $20^{\circ} \mathrm{C}$ in this experiment).

275 red star on the liquid solid line identifies where starting at 40 $276{ }^{\circ} \mathrm{C}$ would relate to $20{ }^{\circ} \mathrm{C}$ for $1: 1$ co-crystal, e.g., as defined by 277 the detail of the component solubility and eutectics. Gradual 278 cooling shows the formation of co-crystals 2:1 from BZ:INA 279 (1:1) in 50\% ethanol, and cooling indicates that the $2: 1$ product 280 is kinetically favored over the 1:1 co-crystal.

281 This was confirmed by examining the powder X-ray 282 diffraction pattern of the solid formed during crystallization 283 with and without seeding. These studies indicate that co284 crystals 2:1 were grown when the crystals were left to grow for $2851 \mathrm{~h}$, the formation of co-crystals $2: 1$ were kinetically favored, 286 and there was no effect of $1: 1$ seeds to enhance the growth of 287 co-crystal 1:1 instead of 2:1, but when the crystals were left to 288 grow over a longer period, only formation of the 1:1 co-crystals 289 occurred. This indicates the 1:1 co-crystals were thermody290 namically favored. It is for this reason that the isothermal 291 ternary phase diagrams have been redrawn to indicate meta stability (Figures 7 and 8), as indicated by the dash line in the 292 upper most portions of the phase regions for 1:1 only, 293 concurrent 1:1 with $2: 1$ and $2: 1$ only. The drawn out cooling 294 crystallization in 50\% ethanol shows the formation of co- 295 crystals 2:1 from BZ:INA (1:1) in 50\% ethanol, and this 296 indicates that this product is kinetically favored over the 1:1 297 system.

298

Starting with the physical mixture of BZ:INA (1:1) the 299 monitoring of crystallization confirms the previous report that 300 only 2:1 co-crystals were grown in water and only $1: 1$ co- 301 crystals were grown in ethanol. ${ }^{5}$ When the physical mixture of 302 BZ:INA was set at a 2:1 stoichiometric ratio, and the solvent 303 composition was varied, the crystal screening clearly showed 304 that an increase in the formation of 2:1 co-crystals was noted as 305 the fraction of water increased, and an increase in 1:1 co- 306 crystals was noted as the fraction of ethanol was increased. This 307 leads to the possibility of tuning the crystallization outcome 308 through the selection of an appropriate solvent composition. 309

To highlight this tuneability opportunity for the crystal- 310 lization on solvent composition, the phase outcome was 311 monitored, and the solution composition was varied from pure 312 water to pure ethanol for a batch cooling crystallization run. 313 Typical outcomes are presented in Figures 9 and 10. As the 314 f9f10

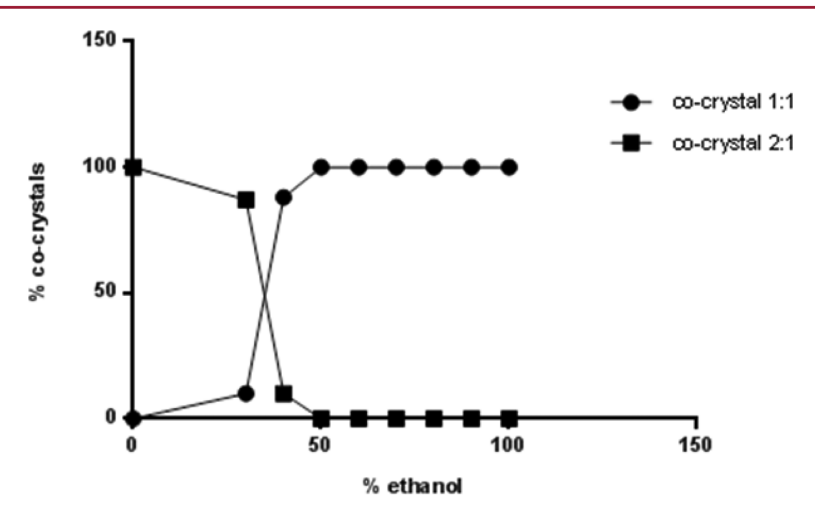

Figure 9. Change in the growth of co-crystals (1:1) and (2:1) from BZ:INA (1:1) with the change of the solvent.

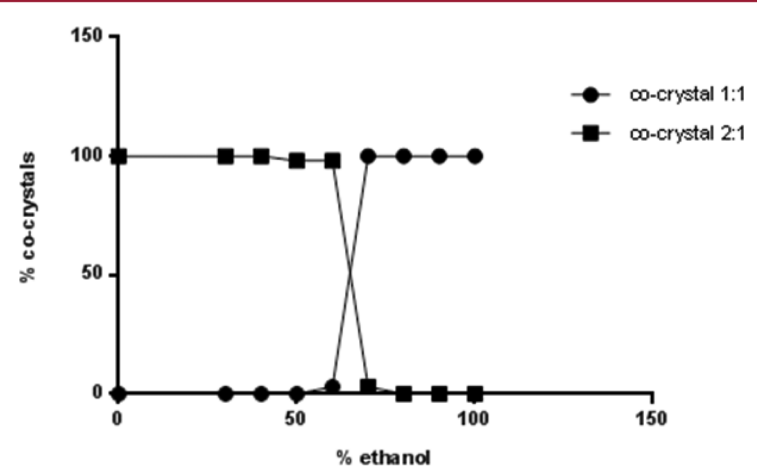

Figure 10. Change in the growth of co-crystals (1:1) and (2:1) from BZ:INA (2:1) with the change of the solvent.

solvent mixture being varied with either a $1: 1$ or $2: 1$ compound 315 ratio being set, the dominate phase around $30 \%$ and $40 \% 316$ respectively for the ethanol mixed solvent was the $2: 1$ system, 317 and for 60 to $100 \%$ the dominant phase was $1: 1$ only. 318

Initially the amount of BZ:INA 1:1 clearly varied as the 319 solvent composition was varied (Figure 9). The curve profile 320 shows that only co-crystals 2:1 were grown in water and only 321 co-crystals 1:1 were grown in ethanol. The growth of $1: 1$ co- 322 
323 crystals started in $30 \%$ ethanol and increased as the growth of 324 2:1 co-crystals decreased with an increase in the concentration 325 of ethanol; however at $60 \%$ only 1:1 co-crystals formed.

326 The curve profile (Figure 10) shows that in water only 2:1 327 co-crystals were grown and only 1:1 in ethanol. At 50\% ethanol 328 there was significant growth of the 1:1 co-crystals, and with an 329 increase in concentration of ethanol this increased and the 330 amount of $2: 1$ co-crystals decreased. In $100 \%$ ethanol only $1: 1$ 331 cocrytals were grown and at $60 \%$ both $1: 1$ and $2: 1$ were most 332 significant.

333 With the seeding studies undertaken, to disrupt inter 334 conversion, the following comparative trends without and 335 with seeding were noted. With the crystallization configured for 336 1:1 formation, this phase was observed after $1 \mathrm{~h}$. However, 337 repeating with seeds of 1:1 co-crystals the initial appearance of $3382: 1$ as a transient phase was reduced, and conversion to 339 complete 1:1 co-crystals was within 5-10 min. With the 340 crystallization configured for $2: 1$ formation, this phase was 341 observed after $1 \mathrm{~h}$, and there was no effect of $1: 1$ seeds to 342 enhance the growth of the 1:1 co-crystal, over the 2:1 within 343 this period.

\section{CONCLUSION}

345 The solubility of both $1: 1$ and 2:1 co-crystals increased, with an 346 increase in concentration of ethanol and with an increase in 347 temperature. The solubility of the $2: 1$ co-crystal is greater than 348 the 1:1 co-crystal in the ethanol-water mixtures, in water the 349 solubility is identical, and at higher ethanol concentration the 350 solubility is similar. Overall, the solubility of $1: 1$ and $2: 1$ co351 crystals was lower than the solubility of the individual 352 components.

353 Further, this study indicates that the solubility of the $1: 1$ and 354 2:1 co-crystals in the mixed solvents was fitted to the general AJ 355 model cosolvency, and the overall fit was within an MPD of $35620 \%$; the model does not really work for this class of 357 compounds. Subsequently, fitting to these studies specific 358 data a MPD of less than $10 \%$ was achieved. In fact, two classes 359 of solubility appear to be present, and which grouping they 360 belong to was found to be dependent on temperature and 361 solvent composition. This was further highlighted by examining 362 the deviation of the actual solubility from the ideal solubility. 363 Such deviation suggests that there is reconfiguration in solute364 solvent interaction during formation of molecular aggregates or 365 the formation of specific modes of molecular complexation, as 366 seen in the solid state. The van't Hoff equation was used to 367 calculate the enthalpy and entropy of solution; the results show 368 that the enthalpy of solution change for the $1: 1$ co-crystals is 369 higher than that of the 2:1 co-crystals, indicating that the $1: 1$ 370 co-crystal has more interaction between the solute and the 371 solvent. The entropy of solution change in 1:1 co-crystal is 372 higher than that for 2:1 co-crystal, indicating that the system for 373 the $1: 1$ co-crystal is more disordered than the $2: 1$ co-crystals. 374 Therefore, 1:1 co-crystals in the solution state are less stable 375 than that of the 2:1 co-crystals. This further substantiates the 376 role of solvent sensitization on the outcome of co-crystal 377 formation.

378 The determination of the solubility curves was an important 379 method to differentiate and identify the polymorphic aspect of 380 this system. From the synthesis perspective of co-crystals for 381 this system, opting for water, ethanol, and mixed solvent 382 impacted the formation of pure $1: 1$ and $2: 1$ co-crystals, or a 383 mixture from both depending on the solvent composition. 384 These findings clearly support the view that the choice of mixed solvent composition influences the step cooling crystallization 385 process significantly, and undertaking ternary phase diagram at 386 two temperatures which define initial and final point on step 387 cooling profile reveals the complex crystallization behavior of 388 1:1 or $2: 1$ co-crystal systems.

389

For the key crystallization parameters, solubility, induction 390 time, and under cooling, the impact of solvent composition has 391 been demonstrated to go beyond composition of BZ:INA 1:1 392 and 2:1 obtained but also impacts on the induction time for the 393 crystallization to occur. For instance when the solvent was 394 water, a notable induction time was seen; however, there was a 395 significant decrease in undercooling at 30\% ethanol, with the 396 associated observation that the temperature of crystallization 397 was lower in water than ethanol, but of a similar magnitude 398 irrespective of solvent composition. Critically, such outcomes 399 do suggest these patterns with crystallization parameters do 400 trend with the picture of mixed modes of association and 401 solvation noted from the solubility screen. 402

To conclude, this contribution reveals the complexity of co- 403 crystal formation, for systems with 1:1 and 2:1 co-crystals, and 404 the way in which solvent composition, along with ternary phase 405 diagrams and solubility studies when combined as presented, 406 supports the rational design of the co-crystals crystallization. 407 Future work will be focused on the solution speciation and the 408 impact such speciation has upon the nucleation process for this 409 type of co-crystal system.

\section{ASSOCIATED CONTENT}

410

\section{Supporting Information}

The Supporting Information is available free of charge on the 413 ACS Publications website at DOI: 10.1021/acs.cgd.5b00908. 414

Van't Hoff equation; the log-linear model of Yalkowsky; 415 the Jouyban-Acree model; phase diagram experimental 416 (PDF)

\section{AUTHOR INFORMATION}

\section{Corresponding Author}

*E-mail: tmunshi@lincoln.ac.uk.

Notes

The authors declare no competing financial interest.

\section{ACKNOWLEDGMENTS}

This work was supported by Pfizer.

\section{REFERENCES}

(1) ter Horst, J. H.; Deij, M. A.; Cains, P. W. Cryst. Growth Des. 2009, 426 9, 1531 .

(2) Pudipeddi, M.; Serajuddin, T. M. J. Pharm. Sci. 2005, 94, 929.428

(3) Blagden, N.; Davey, R. J. Cryst. Growth Des. 2003, 3, 873.

(4) Blagden, N.; Berry, D. J.; Parkin, A.; Javed, H.; Ibrahim, A.; 430 Gavan, P. T.; De Matos, L. L.; Seaton, C. C. New J. Chem. 2008, 32, 431 1659.

(5) Seaton, C. C.; Parkin, A.; Wilson, C. C.; Blagden, N. Cryst. 433 Growth Des. 2009, 9, 47.

(6) Chiarella, R. A.; Davey, R. J.; Peterson, M. L. Cryst. Growth Des. 435 2007, 7, 1223-1226.

(7) Jouyban, A.; Chew, N.; Chan, H.; Sabour, M.; Acree, W. E., Jr. 437 Chem. Pharm. Bull. 2005, 53, 634.

(8) Soltanpour, S.; Acree, J. Biomed. Int. 2010, 1, 9.

(9) Acree, J. J. Pharm. Pharmaceut. Sci. 2008, 11, 32.

(10) Jouyban, A.; Panahi-Azar, V.; Khonsari, F. J. Mol. Liq. 2011, 160, 44 14.

(11) Jouyban, A.; Acree, W. J. Mol. Liq. 2008, 142, 158.

\section{2} 443 
444 (12) Jouyban, A. J. Pharm. Sci. 2008, 11, 32.

445 (13) Acree, W. E. Thermochim. Acta 1992, 198, 71.

446 (14) Boyd, S.; Chadwick, K.; Back, K.; Davey, R. j.; Seaton, C. C. J. 447 Pharm. Sci. 2010, 99, 3779.

448 (15) Prosim Ternary Phase Diagram software (http://www.prosim. 449 net/en/resources/download.html).

450 (16) Ulrich, J.; Jones, M. Chem. Eng. Res. Des. 2004, 82, 1567.

451 (17) Huppert, H. E.; Sparks, R. S. J.; Wilson, J. R.; Hallworth, M. A. 452 Earth Planetary Sci. Lett.; Elsevier Science Publishers B.V.: 453 Amsterdam1986; pp 319-328. 